\title{
Virucidal effect of povidone iodine on COVID-19 in the nasopharynx: an open-label randomized clinical trial
}

\author{
Mostafa Kamal Arefin ${ }^{1}$ (D) S. K. Nurul Fattah Rumi ${ }^{1}$ A. K. M. Nasir Uddin ${ }^{1}$. \\ Sultana Sahana Banu' ${ }^{1}$ Mala Khan ${ }^{2}$ - Ahsanul Kaiser ${ }^{3} \cdot$ Joybaer Anam Chowdhury $^{4}$. \\ Md. Abdullah Saeed Khan ${ }^{5}$ Mohammad Jahid Hasan ${ }^{5}$
}

Received: 14 April 2021 / Accepted: 10 May 2021/Published online: 18 May 2021

(C) Association of Otolaryngologists of India 2021

\begin{abstract}
Povidone-iodine (PVP-I) is a time-tested antiseptic agent with excellent virucidal (99.99\%) properties. Repurposing it against coronavirus disease-19 (COVID-19) is a relatively newer concept and has been sparsely tested in vivo. The most common route of entry of severe acute respiratory syndrome coronavirus 2 (SARS CoV-2) is the nasopharynx. Averting colonization of the virus could be one of the best options to reduce the incidence of infection. PVP-I gargle and mouthwash were found to be effective in vitro rapid inactivation against SARS-CoV-2 on a smaller scale (Hassandarvish et al. in BDJ 1-4, 2020, Pelletier et al. in ENTJ 1-5, 2020). However, efficacy in humans is lacking. To assess the virucidal effect of PVP-I against SARS-CoV-2 located in the nasopharynx was the objective of this parallel armed randomized clinical trial. We screened all RT-PCR-confirmed COVID-19 cases aged 18 years and above with symptoms. Written informed consent was obtained before
\end{abstract}

Mostafa Kamal Arefin

arefin61dmc@gmail.com

S. K. Nurul Fattah Rumi rumi17dr@gmail.com

A. K. M. Nasir Uddin

dmch@hospi.dghs.gov.bd

Sultana Sahana Banu

sultana_aus@yahoo.com

Mala Khan

malakhan_07@yahoo.com

Ahsanul Kaiser

ahsanulkaisertamal@gmail.com

Joybaer Anam Chowdhury

joybaer6106@yahoo.com randomization. Nasopharyngeal clearance of SARS-CoV-2 was tested after single time application of PVP-I nasal irrigation (NI) at diluted concentrations of $.4 \%, .5 \%$ and $.6 \%$ and PVP-I nasal spray (NS) at diluted concentrations of $.5 \%$ and $.6 \%$. All groups were compared to the corresponding controls (distilled water). The primary outcome was viral clearance in a repeat RT-PCR (qualitative), and the secondary outcome was the number of adverse events. Final data analysis was performed using the statistical software SPSS (Version 20). A total of 189 confirmed COVID-19 cases were randomized into seven groups: 27 patients in each group. Of all, $159(84.1 \%)$ were male, and $30(15.9 \%)$ were female. We observed a statistically significant proportion of nasopharyngeal clearance with all strengths of PVP-I NI and PVP-I NS compared to the corresponding controls. Additionally, $0.5 \%$ NI was significantly better than $0.5 \% \mathrm{NS}$ for viral clearance $(p=0.018)$ and had the highest nasopharyngeal clearance among all

Md. Abdullah Saeed Khan

abdullahdmc@gmail.com

Mohammad Jahid Hasan dr.jahid61@gmail.com

1 Dhaka Medical College Hospital, Dhaka, Bangladesh

2 Bangladesh Reference Institute of Chemical Measurements (BRICM), Dhaka, Bangladesh

3 Bangladesh Council of Scientific and Industrial Research (BCSIR), Dhaka, Bangladesh

4 National Institute of Cardiovascular Diseases (NICVD), Dhaka, Bangladesh

5 Pi Research Consultancy Center, Dhaka, Bangladesh 
Table 1 Patient Demographics, baseline clinical characteristics and virucidal activity of PVI-P Nasal spray and mouth wash

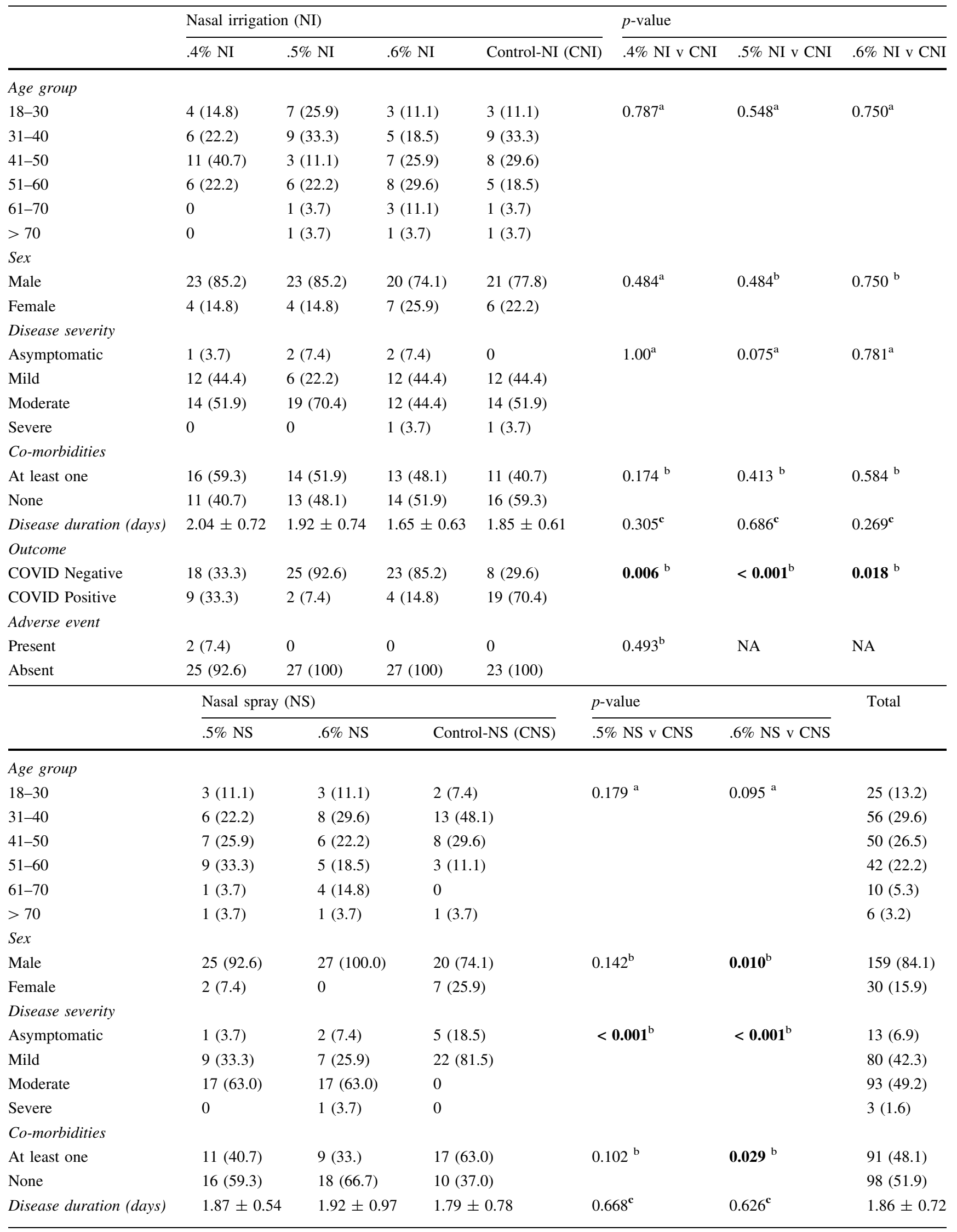


Table 1 continued

\begin{tabular}{|c|c|c|c|c|c|c|}
\hline & \multicolumn{3}{|c|}{ Nasal spray (NS) } & \multicolumn{2}{|l|}{$p$-value } & \multirow[t]{2}{*}{ Total } \\
\hline & $.5 \% \mathrm{NS}$ & $.6 \% \mathrm{NS}$ & Control-NS (CNS) & $.5 \% \mathrm{NS}$ v CNS & $.6 \% \mathrm{NS} \vee \mathrm{CNS}$ & \\
\hline \multicolumn{7}{|l|}{ Outcome } \\
\hline COVID Negative & $18(66.7)$ & $22(81.5)$ & $2(7.4)$ & $<\mathbf{0 . 0 0 1}^{\mathrm{d}}$ & $<0.001^{\mathrm{e}}$ & $116(61.4)$ \\
\hline COVID Positive & $9(33.3)$ & $5(18.5)$ & $25(92.6)$ & & & $73(38.6)$ \\
\hline \multicolumn{7}{|l|}{ Adverse event } \\
\hline Present & 0 & 0 & 0 & NA & NA & $2(1.1)$ \\
\hline Absent & $27(100)$ & $27(100)$ & 27 (100) & & & 185 (98.9) \\
\hline
\end{tabular}

Bold indicates significant ' $p$ ' value $<0.05 \%$

Comorbidities included Diabetes Mellitus, Hypertension, Bronchial Asthma and Ischemic Heart Disease

Outcome was defined as RT-PCR negativity for COVID after intervention

Only adverse event was 'Irritation'

${ }^{a} p$-value was determined by Fisher Exact Test

${ }^{\mathrm{b}}$ Chi-square Test

${ }^{\mathrm{c}}$ Independent Samples $t$ Test

${ }^{\mathrm{d}}$ Multivariate Logistic Regression adjusting for Severity

${ }^{\text {e}}$ Severity and Comorbidity

strengths ( $\mathrm{n}=25,92.6 \%) .0 .6 \% \mathrm{NS}$ is better than CNS and $0.5 \% \mathrm{NS}$ in viral clearance. The only adverse event was nasal irritation recorded in two patients each in the $0.4 \%$ and $0.6 \%$ PVP-I NI groups (Tables 1 and 2). PVP-I NI and NS are proved as effective virucidal agent against SARS$\mathrm{CoV}-2$ in human body. Our recommendation is to use PVP-I in naopharynx (as well as oropharynx) to prevent COVID-19.

Keywords COVID-19 · Povidone Iodine · Nasal spray · Nasal Irrigation

\section{Introduction}

Povidone-iodine (PVP-I) is a time-tested antiseptic agent with excellent virucidal (99.99\%) properties. Repurposing it against coronavirus disease-19 (COVID-19) is a relatively newer concept and has been sparsely tested in vivo. The most common route of entry of severe acute respiratory syndrome coronavirus 2 (SARS CoV-2) is the nasopharynx. Hence, it was targeted to prevent access and contamination of the nasopharynx by several protective measures, including facemasks. Averting colonization of the virus could be one of the best options to reduce the incidence of infection. PVP-I gargle and mouthwash were found to be effective in vitro rapid inactivation against SARS-CoV-2 on a smaller scale $[1,2]$. However, efficacy in humans is lacking. To assess the virucidal effect of PVPI against SARS-CoV-2 located in the nasopharynx was the objective of this parallel armed randomized clinical trial.

\section{Methods}

This single-center, open-label randomized clinical trial with a 7-arm parallel-group design was approved by the ethical review committee of Dhaka Medical College, Bangladesh. (NCT Identifier number: NCT04549376). We screened all reverse transcription-polymerase chain reaction (RT-PCR)-confirmed COVID-19 cases aged 18 years and above with symptoms. Written informed consent was obtained before randomization. Nasopharyngeal clearance of SARS-CoV-2 was tested after single time application of PVP-I nasal irrigation (NI) at diluted concentrations of $0.4 \%, 0.5 \%$ and $0.6 \%$ and PVP-I nasal spray (NS) at diluted concentrations of $0.5 \%$ and $0.6 \%$. All groups were compared to the corresponding controls (distilled water). The primary outcome was viral clearance in a repeat RTPCR (qualitative), and the secondary outcome was the number of adverse events. Final data analysis was performed using the statistical software SPSS (Version 20). The authors followed the Extension of the Consolidated Standards of Reporting Trials (CONSORT) 2010 guideline [3]. The study protocol was published elsewhere [4].

\section{Results}

A total of 189 confirmed COVID-19 cases were randomized into seven groups: 27 patients in each group. All of them completed the study. Of all, 159 (84.1\%) were male, and $30(15.9 \%)$ were female. The average age of the participants was $43.98 \pm 12.67$ years $(\mathrm{SD})$, with the majority 


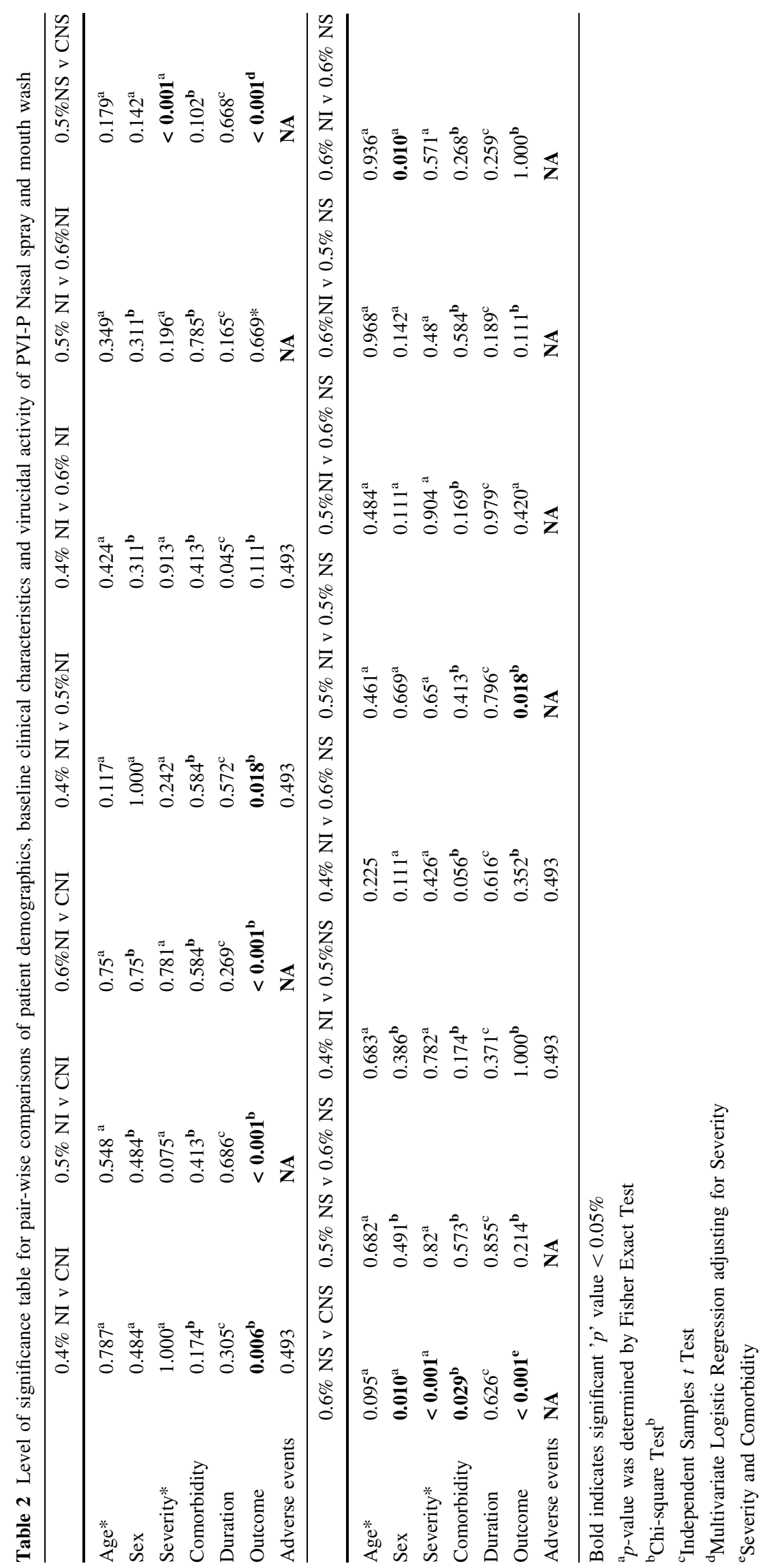


ranging from 31 to 40 years $(\mathrm{n}=56,29.60 \%)$. The PVP-I NS group had significantly higher proportions of severe patients than the control-NS (C-NS) group $(p<0.001)$. The $0.6 \%$ NS group differed from the C-NS group by sex and the presence of comorbidities $(p<0.05)$. Adjusting the differences, we observed a statistically significant proportion of nasopharyngeal clearance with all strengths of PVPI NI and PVP-I NS compared to the corresponding controls. Additionally, $0.5 \%$ NI was significantly better than $0.5 \%$ NS for viral clearance $(p=0.018)$ and had the highest nasopharyngeal clearance among all strengths $(\mathrm{n}=25,92.6 \%)$ and $0.6 \%$ NS is best among $0.5 \%$ NS and CNS. The only adverse event was nasal irritation recorded in two patients each in the $0.4 \%$ and $0.6 \%$ PVP-I NI groups (Tables 1 and 2).

\section{Discussion}

Although in vitro testing of PVP-I solution revealed a considerable reduction in SARS-CoV-2 viral titers, its effect on humans was unclear [1]. Our study confirms the presumed effectiveness of PVP-I in nasopharyngeal clearance of SARS-CoV-2 among COVID-19 patients. Compared to controls, we noted viral clearance at two-fifth to three-fifth dilutions of PVP-I nasal irrigation and spray. Our findings agree with the first report of in vivo PVP-I mouthwash application [5]. In contrast, Guenezan et al. [6] observed no effect of PVP-I on nasopharyngeal viral loads. They reported unpleasant nasal tingling and transient elevation of thyroid stimulating hormone after povidone iodine administration. In contrast, we found nasal irritation in only two patients $(1.1 \%)$. Our study's strength was testing different strengths of PVP-I nasal solution and comparing those with distilled water controls in a modest number of cases. However, our limitation was the inability to conduct viral quantification before and after intervention.

\section{Conclusion}

In this study, a statistically significant proportion of nasopharyngeal clearance with all strengths of PVP-I NI and PVP-I NS (compared to the corresponding controls) is observed, so our recommendation is to use PVP-I prophylactically in nasopharynx as well as oropharynx (through which areas SARS-CoV-2 enters and resides for a while) for prevention of COVID-19.

Acknowledgements The authors acknowledge significant support from the Pi Research Consultancy Center, Dhaka, Bangladesh ( www.pircc.org) in overall research activities. Also, thanks to the
BRICM for material support. The authors would like to thank all the clinical staff of the DMCH for their cordial assistance.

Author contributions MKA had full access to all of the data in the study and takes responsibility for the integrity of the data and the accuracy of the data analysis. Conception of the research idea: MKA. Research design: MKA and MJH. Acquisition, analysis, or interpretation of data: All authors. Drafting of the manuscript: All authors. Critical revision of the manuscript: All authors. Statistical analysis: ASK, MKA and MJH. Administrative, technical, or material support: AKMNU, MK and SSB. Supervision: MKA, SKNFR, and MJH. The authors read and approved the final manuscript.

Funding The trial received research partial grant from BRICM.

Data Availability The corresponding author has access to the alltrial information, and the data will be available on reasonable request (contact: dr.jahid61@gmail.com or arefin61dmc@gmail.com).

\section{Declarations}

Conflict of interest The authors declare that they have no competing interests.

Ethics approval The RCT protocol was approved by the Ethical Review Committee of Dhaka Medical College (Memo No: ERCDMC/ECC/2020/93) on 23 May 2020.

Trial registration The trial protocol has been registered at ClinicalTrials.gov on September 16, 2020. NCT Identifier Number: NCT04549376. (https://clinicaltrials.gov/ct2/show/NCT04549376).

\section{References}

1. Hassandarvish P, Tiong V, Mohamed NA et al (2020) In vitro virucidal activity of povidone iodine gargle and mouthwash against SARS-CoV-2: implications for dental practice. Br Dent J. https://doi.org/10.1038/s41415-020-2402-0

2. Pelletier JS, Tessema B, Frank S, Westover JB, Brown SM, Capriotti JA (2020) Efficacy of povidone-iodine nasal and oral antiseptic preparations against severe acute respiratory syndromecoronavirus 2 (SARS-CoV-2). Ear Nose Throat J. https://doi.org/10.1177/0145561320957237

3. Juszczak E, Altman DG, Hopewell S, Schulz K (2019) Reporting of multi-arm parallel-group randomized trials: extension of the CONSORT 2010 statement. JAMA 321(16):1610-1620. https://doi.org/10.1001/jama.2019.3087

4. Hasan MJ, Rumi SKNF, Banu SS, Uddin AKMN, Islam MS, Arefin MK (2021) Virucidal effect of povidone iodine on COVID19 in the nasopharynx: a structured summary of a study protocol for an open-label randomized clinical trial. Trials 22:2. https://doi.org/10.1186/s13063-020-04963-2

5. Lamas LM, Dios PD, Rodríguez MTP et al (2020) Is povidone iodine mouthwash effective against SARS-CoV-2? First in vivo Tests Oral Dis. https://doi.org/10.1111/odi.13526

6. Guenezan J, Garcia M, Strasters D et al (2021) Povidone iodine mouthwash, gargle, and nasal spray to reduce nasopharyngeal viral load in patients with COVID-19. JAMA-Otolaryngol Neck Surg 9(6):1871. https://doi.org/10.1001/jamaoto.2020.5490

Publisher's Note Springer Nature remains neutral with regard to jurisdictional claims in published maps and institutional affiliations. 